\section{International Scientific Journal Theoretical \& Applied Science}

p-ISSN: 2308-4944 (print) e-ISSN: 2409-0085 (online)

Year: $2015 \quad$ Issue: 12 Volume: 32

Published: $30.12 .2015 \quad$ http://T-Science.org
Nikolay Vasilevich Penshin candidat of economic sciences, Head of the Department "Organization of transportation and road safety" Tambov state technical University avtobd@mail.ru

Victor Yurievich Ivlev Undergraduate, Mechanical engineer Aor Nekrasovskoe RTP, city of Rasskazovo victor.pshenihniy_7@mail.ru

SECTION 27. Transport.

\title{
DETERMINATION OF EMISSIONS OF HARMFUL SUBSTANCES BY MOTOR TRANSPORT
}

Abstract: This article describes the method of field survey of pollutant emissions from road transport in the city of Tambov on the street.

Key words: Transport, emissions, research.

Language: Russian

Citation: Penshin NV, Ivlev VY (2015) DETERMINATION OF EMISSIONS OF HARMFUL SUBSTANCES BY MOTOR TRANSPORT. ISJ Theoretical \& Applied Science 12 (32): 58-61.

Soi: http://s-o-i.org/1.1/TAS-12-32-11 Doi: crossef http://dx.doi.org/10.15863/TAS.2015.12.32.11

\section{ОПРЕДЕЛЕНИЕ ВЫБРОСОВ ВРЕДНЫХ ВЕЩЕСТВ АВТОТРАНСПОРТОМ}

Аннотация: В статье рассматривается метод натурного обследования выбросов загрязняющих веществ от автотранспорта в городе Тамбове на ул. Советской.

Ключевые слова: Автотранспорт, выбросы, исследование.

\section{УДК 502.7}

В период с 8:00 до 18:00 4 ноября 2015 года было проведено исследование автотранспортного потока на перекресте от улицы Московской до улицы М. Горького.
Ширина проезжей части - 19 м.

Количество полос 6.

Ширина разделительной полосы - 0,5 м.

Учет автотранспортных средств.

Таблица 1

\begin{tabular}{|c|c|c|c|c|c|c|c|c|c|}
\hline \multirow[t]{2}{*}{ Дата } & \multirow{2}{*}{$\begin{array}{c}\text { Время } \\
\text { подсчета, за } \\
\text { период } 20 \\
\text { минут } \\
\end{array}$} & \multicolumn{5}{|c|}{ Число автомобилей по категории } & \multicolumn{3}{|c|}{$\begin{array}{c}\text { Скорость движения потока, } \\
\text { км/ч }\end{array}$} \\
\hline & & Л & AM & $\Gamma \leq 12$ & $\Gamma<12$ & $A>3,5$ & $\begin{array}{c}\text { легко- } \\
\text { вые }\end{array}$ & грузовые & автобусы \\
\hline 04.11 .15 & $\begin{array}{l}8 ч 00 \mathrm{M} .- \\
8 ч 20 \mathrm{M}\end{array}$ & 1015 & 0 & 0 & 0 & 98 & 60 & 0 & 50 \\
\hline 04.11 .15 & $\begin{array}{l}8 ч 20 \text { м.- }^{-} \\
8 ч 40 \mathrm{M}\end{array}$ & 1098 & 0 & 0 & 0 & 95 & 70 & 0 & 50 \\
\hline 04.11 .15 & $\begin{array}{l}\text { 8ч40м.- } \\
9 ч 00 \mathrm{M}\end{array}$ & 1314 & 0 & 0 & 0 & 102 & 60 & 0 & 60 \\
\hline 04.11 .15 & $\begin{array}{l}17 ч 00 \mathrm{M} .- \\
17 ч 20 \mathrm{M}\end{array}$ & 983 & 0 & 0 & 0 & 92 & 70 & 0 & 60 \\
\hline 04.11 .15 & $\begin{array}{l}17 ч 20 \mathrm{M} .- \\
17 ч 40 \mathrm{M}\end{array}$ & 1005 & 0 & 0 & 0 & 103 & 60 & 0 & 60 \\
\hline 04.11 .15 & $\begin{array}{l}17 ч 40 \mathrm{M} .- \\
18 ч 00 \mathrm{M}\end{array}$ & 1452 & 0 & 0 & 0 & 114 & 60 & 0 & 50 \\
\hline
\end{tabular}




\begin{tabular}{l|lrl|l|ll} 
& ISRA (India) & $=\mathbf{1 . 3 4 4}$ & SIS (USA) & $=\mathbf{0 . 9 1 2}$ & ICV (Poland) & $\mathbf{0 . 6 3 0}$ \\
Impact Factor: & ISI (Dubai, UAE) $=\mathbf{0 . 8 2 9}$ & PUHL (Russia) $=\mathbf{0 . 1 7 9}$ & PIF (India) & $=\mathbf{1 . 9 4 0}$ \\
& GIF (Australia) & $\mathbf{0 . 5 6 4}$ & ESJI (KZ) & $=\mathbf{1 . 0 4 2}$ & & \\
JIF & $\mathbf{1 . 5 0 0}$ & SJIF (Morocco) $=\mathbf{2 . 0 3 1}$ & & \\
\end{tabular}

Методика определения выбросов автотранспорта для проведения сводных расчетов загрязнения атмосферы городов использовалась при проведении исследовательской работы.

Выброс і-того загрязняющего вещества (г/с) движущимся автотранспортным потоком на автодороги (или её участке) с фиксированной протяженностью L (км) определяется по формуле:

$$
M_{L 1}=\frac{L}{1200} \sum_{1}^{k} M_{k i}^{L} \cdot G_{k} \cdot r_{V k i},(\text { г/c })
$$

где $M_{k i}^{L}(г / \kappa м)-$ удельный пробеговый выброс iго вредного вещества автомобилями k-ой группы для городских условий эксплуатации;

$k$ - количество групп автомобилей;

$\mathrm{Gk}$ - фактическая наибольшая интенсивность движения;

$r_{V k i^{-}}$поправочный коэффициент, учитывающий среднюю скорость движения транспортного потока $\left(\cdot r_{V k i}\right.$, км/час) на выбранной автомагистрали (или её участке);

$L$ (км) - протяжённость автодороги (или её участка).

$$
\begin{aligned}
& M_{L 1}=\frac{250}{1200} 2,3 \cdot 3720 \cdot 0,75=1336,875 \Gamma / \mathrm{c} \\
& M_{L 2}=\frac{230}{1200} 2,3 \cdot 3720 \cdot 0,75=1229,925 \Gamma / \mathrm{c}
\end{aligned}
$$

Под расчётной оценкой уровня загрязнения воздуха в зонах перекрестков следует понимать наибольшее значение содержания вредных веществ в отработавших газах. Выброс і-го загрязняющего вещества в зоне перекрёстка при запрещающем сигнале светофора определяется по формуле:

$$
M_{\Pi i}^{3}=\frac{P_{u}}{60} \sum_{1}^{N u} \sum_{1}^{N \Gamma p} M_{\Pi i \kappa}^{\prime} \cdot G_{k}, \text { г/мин }
$$

где $P_{4-}$ продолжительность действия запрещающего сигнала светофора (включая жёлтый цвет), с;

$N_{ц} \quad-\quad$ количество циклов действия запрещающего сигнала светофора за 20минутный период времени;

$N_{z p}$ - количество групп автомобилей;
$M_{\text {Пік }}^{\prime}($ г/мин) -удельный выброс і-го ЗВ автомобилями, k-ой группы, находящихся в «очереди» у запрещающего сигнала светофора;

$G_{k}-$ количество автомобилей $\mathrm{k}$ группы, находящихся в «очереди» в районе перекрёстка в конце каждого цикла действия запрещающего сигнала светофора.

Суммарный разовый выброс і-го загрязняющего вещества (г/с) автотранспортом в одном направлении движения за 20- минутный период дополнительного обследования в районе перекрестка определяется по формуле:

$$
\begin{gathered}
M_{\Pi i}^{c}=\frac{1}{1200}\left(M_{\Pi i}^{3}+M_{L i}^{p}\right), \Gamma / \mathrm{c} \\
M_{L i}^{p}=L^{\Pi^{\prime}} \sum_{1}^{N^{\prime} u} \sum_{1}^{N \Gamma p} M_{k i}^{L} \cdot G_{k p} \cdot r_{V k i}, \Gamma / \mathrm{c}
\end{gathered}
$$

где $L^{\Pi}$ - расстояние, проходимое автомобилями в одном направлении при разрешающих сигналах светофора в течение 20 минут, состоящее из длины очереди автомобилей, образуемой при запрещающем сигнале светофора, и длины соответствующей зоны перекрестка, км;

$N^{\prime} u$ - количество циклов работы разрешающего сигнала светофора в течение 20 минут;

$G_{k p}$ - количество автомобилей каждой k-ой категории, проходящих через зону перекрёстка в одном направлении при разрешающем сигнале светофора.

При необходимости оценки общего разового выброса і-го загрязняющего вещества (г/с) автотранспортом в районе перекрестка суммируются разовые выбросы по каждому направлению движения.

$$
\begin{aligned}
& M_{\Pi i}^{3}=\frac{20}{60}(2,3 \cdot 1142+14,48 \cdot 98)=1348,547 \text { г/мин } \\
& M_{L i}^{p}=80(2,3 \cdot 3720 \cdot 0,75)=513360 \text { г/с } \\
& M_{\Pi i}^{c}=\frac{1}{1200}(1348,547+513360)=428,9238 \text { г/с. } \\
& \text { В качестве исходных данных для расчета }
\end{aligned}
$$
выбросов автотранспорта в атмосферу использовались результаты натурных обследований структуры и интенсивности автотранспортных потоков с разделением по основным категориям автотранспортных средств. 


\begin{tabular}{l|lrl|l|ll} 
& ISRA (India) & $=\mathbf{1 . 3 4 4}$ & SIS (USA) & $=\mathbf{0 . 9 1 2}$ & ICV (Poland) & $=\mathbf{6 . 6 3 0}$ \\
Impact Factor: & ISI (Dubai, UAE) $=\mathbf{0 . 8 2 9}$ & PUHU (Russia) $=\mathbf{0 . 1 7 9}$ & PIF (India) & $=\mathbf{1 . 9 4 0}$ \\
& GIF (Australia) & $\mathbf{0 . 5 6 4}$ & ESJI (KZ) & $=\mathbf{1 . 0 4 2}$ & & \\
& JIF & $=1.500$ & SJIF (Morocco) & $=\mathbf{2 . 0 3 1}$ & &
\end{tabular}

Таблица 2

Интенсивность движения автомобилей на улице Советская, от улицы Московской до улицы М. Горького с 8.00 до 9.00 04.11.2015 г.

\begin{tabular}{|c|c|c|c|c|c|}
\hline \multirow[t]{2}{*}{ Тип автомобиля } & \multicolumn{3}{|c|}{ Кол-во автомобилей } & \multirow{2}{*}{$\begin{array}{c}\text { Среднее количество } \\
\text { автомобилей за } 20 \\
\text { минут }\end{array}$} & \multirow{2}{*}{$\begin{array}{c}\text { Кол-во } \\
\text { автомобилей за } \\
\text { час } \\
\end{array}$} \\
\hline & 20 минут & 20 минут & 20 минут & & \\
\hline Легкий грузовой & 0 & 0 & 0 & 0 & 0 \\
\hline Средний грузовой & 0 & 0 & 0 & 0 & 0 \\
\hline Тяжелый грузовой & 0 & 0 & 0 & 0 & 0 \\
\hline Легковой & 1013 & 1098 & 1314 & 1142 & 3425 \\
\hline Автобус & 98 & 95 & 102 & 98 & 295 \\
\hline $\begin{array}{c}\text { Общее количество } \\
\text { автомобилей }\end{array}$ & 1111 & 1193 & 1416 & 1240 & 3720 \\
\hline
\end{tabular}

Таблица 3

Интенсивность движения автомобилей на улице Советская, от улицы Московской до улицы М. Горького с 17.00 до 18.00 04.11.2015 г.

\begin{tabular}{|c|c|c|c|c|c|}
\hline \multirow[t]{2}{*}{ Тип автомобиля } & \multicolumn{3}{|c|}{ Кол-во автомобилей } & \multirow{2}{*}{$\begin{array}{c}\text { Среднее количество } \\
\text { автомобилей за } 20 \\
\text { минут }\end{array}$} & \multirow{2}{*}{$\begin{array}{c}\text { Кол-во } \\
\text { автомобилей за } \\
\text { час } \\
\end{array}$} \\
\hline & 20 минут & 20 минут & 20 минут & & \\
\hline Легкий грузовой & 0 & 0 & 0 & 0 & 0 \\
\hline Средний грузовой & 0 & 0 & 0 & 0 & 0 \\
\hline Тяжелый грузовой & 0 & 0 & 0 & 0 & 0 \\
\hline Легковой & 983 & 1005 & 1452 & 1147 & 3440 \\
\hline Автобус & 92 & 103 & 114 & 103 & 309 \\
\hline $\begin{array}{c}\text { Общее количество } \\
\text { aвтомобилей }\end{array}$ & 1075 & 1108 & 1566 & 1250 & 3749 \\
\hline
\end{tabular}

На рисунке 1 представлены графики загруженности улиц автотранспортом в утренние и вечерние часы «пик».
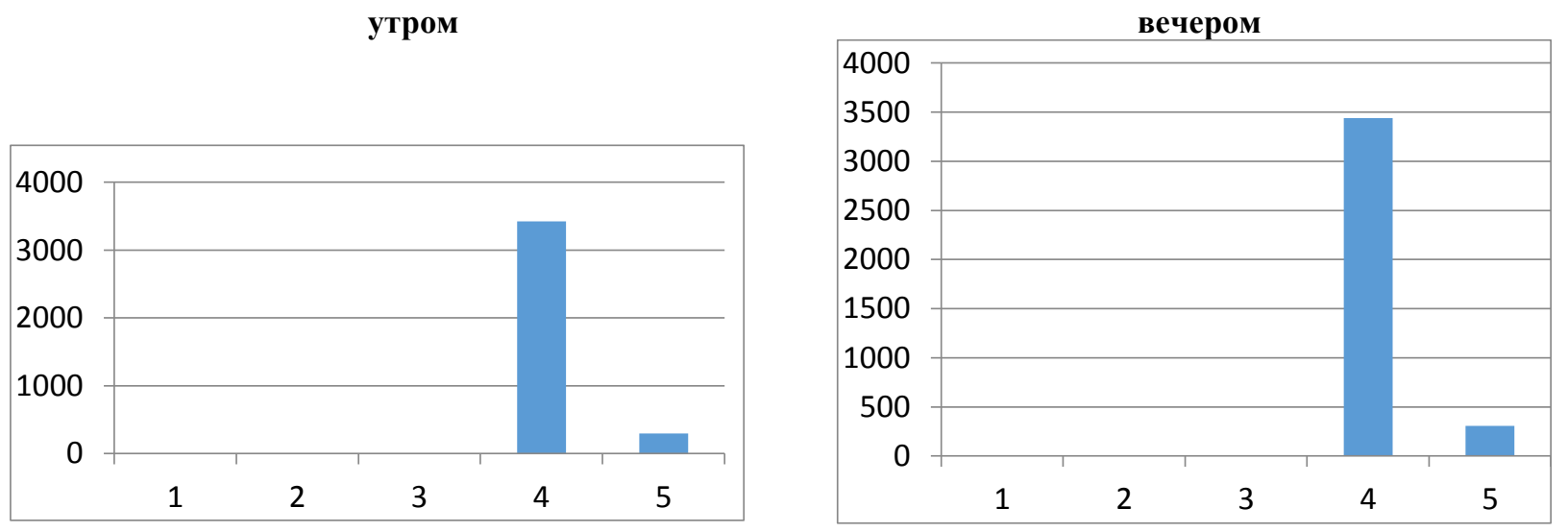

Рисунок 1 - График загруженности улицы автотранспортом (утром и вечером): 1-легкий грузовой; 2- средний грузовой; 3-тяжелый грузовой; 4-легковой; 5- автобус

Проведенный анализ собранных и рассчитанных данных позволил квалифицировать автотранспортный поток, определить группы автотранспорта, оказывающие наибольший вклад в загрязнение атмосферы города (с учетом режима движения автомобильного потока).
Проведенная работа позволит в дальнейшем использовать полученные данные для составления сводных отчетов по выбросам, конкретным расчетам по загрязнению атмосферного воздуха и последующей оценке влияния загрязненного воздуха на население,

ISPC Applied technologies, 


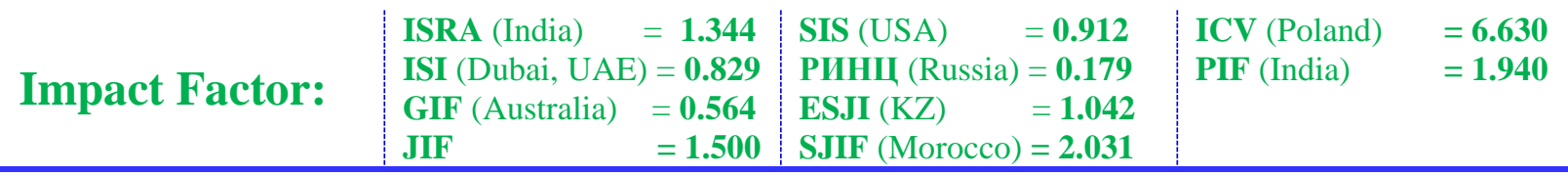

проживающее вдоль городских автомагистралей, a так же дать некоторые рекомендации по организации и упорядочению движения автотранспорта в пределах жилых районов города: создание дополнительных автотранспортных развязок, перенаправление движения грузового транзитного автотранспорта в объезд жилых районов.

\section{References:}

1. Adushkin VV (2001) The ratio of anthropogenic and natural components in the flow of gases in the atmosphere // Global environmental changes. 2001. Novosibirsk: Publishing House of SB RAS, a subsidiary of "Geo", 2001.

2. Evgeniev IE, Karimov BR (1997) High ways and Environment. Proc. - Moscow, 1997.

3. Molodtcov VA (2013) Security vehicles: a tutorial / VA great. - TambovUniv VPO "TSTU», 2013. - 236 p.

4. Molodtcov VA, Guskov AA (2014) Determination of pollutante missions from motor vehicles: method. instructions. TambovUniv VPO "TSTU», 2014. - 22 p.

5. Novikov Y (2002) Ecology, environment and people: Textbook. The manual for high schools, high schools and colleges. Moscow: Fair-Press, 2002. $-506 \mathrm{p}$.
6. Lozhkyn VN, Demochko OI, et al. (1990) Experimental evaluation of the estimated emissions of harmful substances in exhaust gases of internal combustion engines on the operational modes. Technical report on the research. Saint-Petersburg., NGOs CNIT, 1990.

7. (2012) FederalLawof 04.05.1999, № 96-FZ (ed.By 25.06.2012) "OnAirProtection".

8. (2012) Method of determining emissions of harmful substances (pollutants) into the air from road traffic flows, moving on road sof St. Petersburg (app.The order of the Committee on the Environment, Environmental Protection and Ecological Safety of St. Petersburg Government of 17.02.2012 № 23- R).

9. (1997) The environmental problems of road transport. - Moscow, 1997.

10. (1990) Ecological safety of traffic, edited by AB Moscow DyakovVehicles - 1990. 